\title{
Risk of Stroke During Long-Term Anticoagulant Therapy in Patients after Myocardial Infarction
}

\author{
Aida J. Azar, PhD, ${ }^{* \dagger}$ Peter J. Koudstaal, MD, PhD, $\neq$ Axel R. Wintzen, MD, $\$$ Paul F. van Bergen, PhD, $\dagger$ \\ Jan J. Jonker, $\mathrm{MD}, \dagger$ and Jaap W. Deckers, $\mathrm{MD}, \mathrm{PhD}^{*}$
}

Myocardial infarction survivors have an increased risk of stroke, which is reduced with long-term anticoagulant therapy. However, an estimated 10-times increase in risk of bleeding during such treatment has been reported. We evaluated the risk of stroke in patients after a myocardial infarction and examined the relationship of the risk of intracranial hemorrhage or cerebral infarction and the intensity of anticoagulant therapy. The study population consisted of 3,404 post-myocardial infarction patients who took part in a randomized, double-blind, placebo-controlled trial. Patients were randomized to treatment with anticoagulants (international normalized ratio range, $2.8-4.8$ ) or matching placebo. Mean follow-up was more than 3 years. The incidence of stroke analyzed on "intention-to-treat" was 0.7 per 100 patient-years in the anticoagulant patients against 1.2 in placebo, a hazard ratio of 0.60 , with $95 \%$ confidence interval of 0.40 to 0.90. In the anticoagulation group, 15 patients had cerebral infarction and 17 an intracranial bleeding, 3 of which occurred after withdrawal of treatment. In the placebo group, the numbers were 43 and 2 . Of the 14 intracranial bleeds during anticoagulation, 6 occurred at an international normalized ratio between 3.0 and 4.0 and 8 at greater than 4.0. These results confirm that long-term anticoagulant therapy substantially reduces the risk of stroke in postmyocardial infarction patients. The increased risk of bleeding complications associated with anticoagulant therapy is offset by a marked reduction in ischemic events. The risk of intracranial bleeding is directly related to the intensity of anticoagulant treatment.

Azar AJ, Koudstaal PJ, Wintzen AR, van Bergen PF, Jonker JJ, Deckers JW. Risk of stroke during longterm anticoagulant therapy in patients after myocardial infarction. Ann Neurol 1996;39:301-307

Survivors of myocardial infarction have an increased risk of thromboembolic events, including stroke, that has been estimated between $5 \%$ and $8 \%$ in the first year [1]. Administration of long-term anticoagulant therapy may reduce the number of such events [2]. However, an estimated 10-times increase in risk of bleeding during such treatment has been reported [27]. Approximately $50 \%$ of these bleedings are fatal [4]. The presence of hypertension, older age, and trauma has been associated with an increased risk of stroke [6].

The results of the three recently conducted postmyocardial infarction trials indicated a 40 to $55 \%$ reduction in the risk of stroke in patients treated with anticoagulant therapy for 2 to 3 years compared with placebo $[8-10]$. The intensity of anticoagulant therapy in the three trials was targeted at 2.5 to 5.0 international normalized ratio (INR). The number of intracranial bleedings was higher in the anticoagulated group, but cerebral infarctions were more common in the placebo group.

The purpose of this report is to describe the risk of stroke for post-myocardial infarction patients treated with long-term anticoagulant therapy. In addition, we examined the relationship of the risk of intracranial hemorrhage or infarction and the intensity of anticoagulant therapy. The study population comprised 3,404 patients randomized to anticoagulant therapy or matching placebo.

\section{Patients and Methods}

\section{Patients}

We studied post-myocardial infarction patients who took part in the ASPECT (Anticoagulants in the Secondary Prevention of Events in Coronary Thrombosis) trial. This trial has been described earlier [8]. In short, ASPECT was a randomized, double-blind, placebo-controlled, multicenter clini-
From the *Thoraxcenter and Departments of ${ }^{*}$ Cardiology and $\$$ Neurology, University Hospital Rocterdam, †ASPECT Coordinating Centre, Rotterdam; and $\$$ Department of Neurology, University Hospital Leiden, The Netherlands.
Received Dec 2, 1994, and in revised form Jun 12 and Nov 6. Accepted for publication Nov 9, 1995.

Address correspondence to Dr Azar, Department of Clinical Epidemiology, Thoraxcenter BD 377, University Hospital Dijkzigt, Dr Molewaterplein 40, 3015 GD Rotterdam, The Netherlands. 
cal trial that compared anticoagulant therapy with matching placebo on mortality and cardiovascular events in post-myocardial infarction patients.

Hospital survivors of acute myocardial infarction were screened for eligibility just prior to hospital discharge. Patients with an indication for oral anticoagulant therapy, including the presence of atrial fibrillation, left ventricular aneurysm or thrombus, or with clinical overt evidence of heart failure at discharge, were not eligible. Informed consent was obtained in all subjects. Patients were randomly assigned to treatment with oral anticoagulant therapy or to matching placebo. From September 1, 1986, to December 31, 1991, 3,404 patients were randomized. The study population contributed 10,441 patient-years of follow-up.

\section{Oral Anticoagulation and Dose Adjustment}

The target anticoagulant range was 2.8 to 4.8 INR [11-15]. Individual dose adjustments were guided by prothrombin time measurements obtained at regular intervals at specialized anticoagulant clinics, the Thrombosis Centers. Anticoagulant treatment consisted of phenprocoumon, acenocoumarol, or matching placebo tablets. Double-blinding was maintained by conversion of the actual prothrombin time values of placebo-treated patients to fake values within therapeutic range by a computerized dosage algorithm at the anticoagulant clinic.

At each follow-up visit to the anticoagulant clinic, a short history was taken and a blood sample drawn for determination of the prothrombin time. The prothrombin time is the time needed for a blood sample to clot when a small quantity of thromboplastin reagent is added [15]. Patients were seen at the initial (ie, randomization) visit and on a weekly basis thereafter until the prothrombin time measurements were within the specified target range. The interval between visits was subsequently prolonged until a maximum period of 8 weeks. Patients requiring frequent dosage adjustments were seen more regularly. While on trial medication, patients were strongly advised not to take other antithrombotic medication. At the end of the trial in June 1992, trial medication was discontinucd in all patients.

If available, prothrombin time measurements were obtained immediately before the hemorrhagic and nonhemorrhagic episode. Otherwise, the last measurement within a maximum time frame of 28 days prior to the stroke was registered.

\section{Clinical Events}

Information on clinical events was obtained directly from the patients when they visited the Thrombosis Center or from their general practitioners. In case of hospitalization, additional information was retrieved from the hospital records.

Stroke was diagnosed according to internationally accepted criteria for the assessment of cerebrovascular disease [16]. Brief attacks lasting less than 24 hours and leaving no residual symptoms were classified as transient ischemic attacks (TIAs) [16]. If neurological death occurred within 24 hours, a diagnosis of intracranial bleeding was made unless findings on computed tomographic (CT) scanning indicated otherwise. Events lasting beyond 24 hours were specified as cerebral infarction or intracranial bleeding on the basis of CT scanning, if available. If a CT scan was not available, the event was classified as unspecified. The diagnosis and classification of clinical events was made by two neurologists (P.J.K., A.R.W.), who independently reviewed the clinical course of each case on the basis of a standardized patient report. The committee members also registered and coded all neurological admissions. They were blinded to treatment assignment and were not informed of actual prothrombin time measurements. No distinction was made between primary intracerebral hemotrhage and hemorrhagic infarction.

The functional outcome of all cerebrovascular events was classified as survival with no disability, survival with mild disability (residual symptoms, but without impact on daily activities), survival with moderate disability (symptoms significantly interfering with normal daily activities, the patient being unable to live independently), survival with severe disability (patient completely dependent in all activities of daily living), or death. Other neurological nonvascular complications diagnosed in the course of the trial included cerebral tumor (11 patients), Guillain-Barré syndrome, lumbar disk hernia (20 patients), motor neuron disease (11 patients), and polyneuropathy (3 patients).

\section{Data Analysis}

A comparison between the anticoagulated and placebo groups with respect to the incidence of stroke was made in terms of the hazard ratio, ie, the risk of stroke per unit time for patients randomly assigned to oral anticoagulant therapy divided by the risk for those randomly assigned to placebo. Hazard ratios were obtained with the Cox proportional hazards model [17]. The precision of the estimates was described with their $95 \%$ confidence intervals. For "intention-to-treat" analysis, all randomized patients were included irrespective of discontinuation of trial medication. Censoring was applied when the patient died, had a stroke, or at the end of followup, on June 30,1992 . In a subsidiary analysis, only those strokes that occurred while the patient was on trial treatment (or within 28 days after its cessation) were taken into account ("per-protocol" analysis).

\section{Results}

Demographic data, smoking habits, medical history, and medication used during hospitalization are presented in Table 1. The median age of the patients was 61 years and $80 \%$ of the study population were men. As evidenced by their low prevalence of heart failure (only $5 \%$ in Killip class III or IV) at any time during their hospitalization, and a small number $(9 \%)$ with a previous myocardial infarction, ASPECT patients were representative of a relatively low-risk post-myocardial infarction population.

Trial treatment was discontinued in 1,655 patients (768 after anticoagulant therapy and 887 in placebo). Reasons for discontinuation were vascular events ( 485 patients: 215 anticoagulant therapy and 270 placebo) and nonmedical in the 1,170 other patients (553 anti- 
Table 1. Selected Demographic, Baseline, and Randomization Characteristics

\begin{tabular}{|c|c|c|c|c|c|c|}
\hline & \multicolumn{2}{|c|}{ Anticoagulant } & \multicolumn{2}{|c|}{ Placebo } & \multicolumn{2}{|c|}{ Total } \\
\hline & $(\mathrm{n}=1,700)$ & $(\%)$ & $(\mathrm{n}=1,704)$ & $(\%)$ & $(\mathrm{n}=3,404)$ & $(\%)$ \\
\hline \multicolumn{7}{|l|}{ Age $(y r)$} \\
\hline$<55$ & 507 & $(30)$ & 490 & (29) & 997 & (29) \\
\hline $55-65$ & 536 & (32) & 573 & (33) & 1,109 & (33) \\
\hline$>65$ & 657 & (38) & 641 & (38) & 1,298 & $(38)$ \\
\hline Male & 1,370 & $(81)$ & 1,350 & (79) & 2,720 & $(80)$ \\
\hline Previous $\mathrm{MI}^{\mathrm{a}}$ & 157 & (9) & 150 & (9) & 307 & (9) \\
\hline Previous use of $\mathrm{AC}^{\mathrm{a}}$ & 35 & $(2)$ & 44 & $(2)$ & 79 & (2) \\
\hline Hematocrit $(\geq 45)$ & 735 & $(43)$ & 767 & $(45)$ & 1,502 & $(44)$ \\
\hline Killip III/IV & 96 & (5) & 93 & $(5)$ & 189 & $(5)$ \\
\hline History of DM & 134 & (8) & 125 & (7) & 259 & (8) \\
\hline Current smokers & 894 & $(53)$ & 888 & $(52)$ & 1,782 & $(52)$ \\
\hline \multicolumn{7}{|l|}{ Treatment } \\
\hline$\beta$-blockers & 867 & $(51)$ & 869 & $(51)$ & 1,736 & $(51)$ \\
\hline Acetyl salicylic acid ${ }^{b}$ & 118 & (7) & 104 & $(6)$ & 222 & (7) \\
\hline Mean SBP, mm Hg (SD) & 119 & (16) & 119 & $(16)$ & 119 & $(16)$ \\
\hline
\end{tabular}

${ }^{2}$ Prior to the qualifying myocardial infarction.

${ }^{b}$ Discontinued at randomization.

$\mathrm{MI}=$ myocardial infarction; $\mathrm{AC}=$ anticoagulant; $\mathrm{DM}=$ diabetes mellitus; $\mathrm{SBP}=$ systolic blood pressure at discharge; $\mathrm{SD}=$ standard deviation.

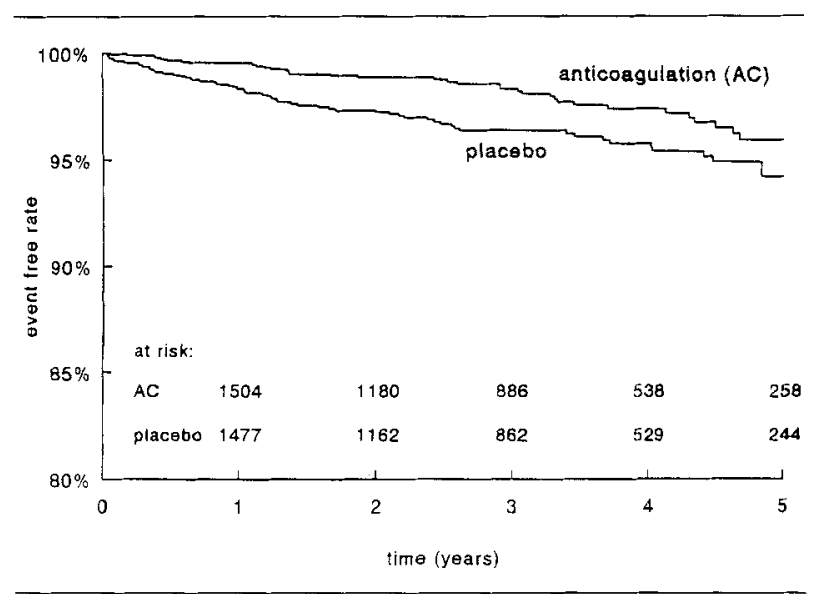

Kaplan-Meier curves for stroke from any cause, according to assigned therapy. Numbers denote patients at risk (anticoagulated patients on top).

coagulant therapy and 617 placebo). For the overall duration of the trial, patients received trial medication for $71 \%$ of the time in the anticoagulated and $67 \%$ in the placebo group.

The time lapse between randomization and onset of stroke, analyzed on an intention-to-treat basis, is presented in the Figure. The duration of treatment ranged from 1 day to 6 years. Three years following randomization, $2 \%$ of the patients on anticoagulant therapy had suffered a stroke compared with $4 \%$ in placebo.
The incidence of stroke analyzed on intention-totreat and per-protocol analysis is presented in Table 2. Based on the total observation time (intention-totreat), 99 patients were diagnosed as having a stroke. The risk of stroke increased with age; patients in the highest age tertile ( $>65$ years) accounted for more than $50 \%$ of all strokes. The incidence of stroke was marginally but not statistically significantly higher in patients with a previous myocardial infarction $(11.1 \%$ vs $8.1 \%)$ and with heart failure (Killip class 3 or 4 ) during hospitalization $(5.5 \%$ vs $4.0 \%$ ), and in patients ( $n=100$ ) developing arrhythmias following discharge $(3.0 \%$ vs $2.9 \%)$. The incidence of stroke was 0.7 per $100 \mathrm{pa}$ tient-years in the anticoagulated group and 1.2 per 100 patient-years in placebo, a hazard ratio (HR) of 0.60 with a $95 \%$ confidence interval (CI) of 0.40 to 0.90 , a $40 \%$ reduction in the risk of stroke in the anticoagulated group.

The risk of hemorrhages was eight times greater for anticoagulated patients compared with placebo. Of the 17 with intracranial bleeding in the anticoagulated group, 15 were diagnosed on the basis of a CT scan (9 intracerebral, 3 subdural, and 3 subarachnoidal). Eight of the 17 bleedings were fatal; 6 were diagnosed by CT scan (3 intracerebral, 2 subdural, and 1 subarachnoidal) and 2 on the basis of death within 24 hours. Of the 2 with intracranial bleeding in the placebo group, 1 was subdural and 1 undefined. No fatal intracranial bleeding occurred in the placebo group. 


\begin{tabular}{|c|c|c|c|}
\hline & $\begin{array}{l}\text { Anticoagulant } \\
(\mathrm{n}=1,700)\end{array}$ & $\begin{array}{l}\text { Placebo } \\
(\mathrm{n}=1,704)\end{array}$ & $\mathrm{HR}(95 \% \mathrm{CI})$ \\
\hline \multicolumn{4}{|l|}{ Intention to treat } \\
\hline Patient-years (py) of follow-up & 5,241 & 5,200 & \\
\hline First stroke & $37(0.7 / 100 \mathrm{py})$ & $62(1.2 / 100$ py) & $0.60(0.4-0.9)$ \\
\hline Intracranial bleeding & 17 & 2 & \\
\hline Fatal & 8 & 0 & \\
\hline Nonfatal & 9 & 2 & \\
\hline Cerebral infarction & 15 & 43 & \\
\hline Fatal & 2 & 2 & \\
\hline Nonfatal & 13 & 41 & \\
\hline Transient ischemic attack & 2 & 6 & \\
\hline Unspecified & 4 & 12 & \\
\hline Fatal & 1 & 6 & \\
\hline Nonfatal & 3 & 6 & \\
\hline \multicolumn{4}{|l|}{ Per protocol } \\
\hline Patient-years (py) of follow-up & 3,725 & 3,488 & \\
\hline First stroke & $24(0.6 / 100$ py $)$ & $42(1.2 / 100$ py $)$ & $0.57(0.34-0.93)$ \\
\hline Intracranial bleeding & 14 & 1 & \\
\hline Fatal & 7 & 0 & \\
\hline Nonfatal & 7 & 1 & \\
\hline Cerebral infarction & 6 & 28 & \\
\hline Fatal & 0 & 2 & \\
\hline Nonfatal & 6 & 26 & \\
\hline Transient ischemic attack & 1 & 6 & \\
\hline Unspecified & 3 & 7 & \\
\hline Fatal & 1 & 1 & \\
\hline Nonfatal & 2 & 6 & \\
\hline
\end{tabular}

ASPECT $=$ Anticoagulants in the Secondary Prevention of Events in Coronary Thrombosis; HR = hazard ratios; CI = confidence intervals.

Table 3. Functional Outcome of Stroke Survivors

\begin{tabular}{|c|c|c|c|c|c|c|c|c|}
\hline & \multicolumn{2}{|c|}{ Bleeding } & \multicolumn{2}{|c|}{ Infarction } & \multicolumn{2}{|c|}{ TIA } & \multicolumn{2}{|c|}{ Unspecified } \\
\hline & $\mathrm{AC}$ & PL & $\mathrm{AC}$ & PL & $\mathrm{AC}$ & $\mathrm{PL}$ & $\mathrm{AC}$ & Pl \\
\hline No. of survivors & 9 & 2 & 13 & 41 & 2 & 6 & 3 & 6 \\
\hline \multicolumn{9}{|l|}{ Disability } \\
\hline None & 2 & 2 & 5 & 11 & 2 & 6 & 2 & 3 \\
\hline Mild & 4 & 0 & 4 & 13 & 0 & 0 & 1 & 1 \\
\hline Moderate & 1 & 0 & 4 & 8 & 0 & 0 & 0 & 1 \\
\hline Severe & 2 & 0 & 0 & 9 & 0 & 0 & 0 & 1 \\
\hline
\end{tabular}

$\mathrm{AC}=$ anticoagulant; $\mathrm{PL}=$ placebo; $\mathrm{TIA}=$ transient ischemic attacks.

The functional outcome of the patients who survived a stroke is indicated in Table 3. Sixty-seven percent of the anticoagulated patients who survived a hemorrhagic bleeding were mildly or not disabled, compared with $100 \%$ for placebo.

The incidence of cerebral infarction was approximately three times higher in the placebo than the anticoagulated group. Sixty-nine percent of the anticoagulated patients who survived an infarction were mildly or not disabled, compared with $59 \%$ for placebo.

Six patients in the anticoagulation and 18 in the placebo group had an unspecified event or TIA. Six fatal events occurred in the placebo group, versus one in the anticoagulation group. All patients in the anticoagulant group who survived an unspecified stroke were mildly or not disabled, compared with $67 \%$ for placebo.

When all strokes were combined, the following figures were obtained: The number of patients who died or were severely disabled amounted to 13 in the anticoagulation group, versus 18 in the placebo group. When moderately disabling stroke was included, these figures were 18 and 27 , respectively.

Among the 24 patients with a stroke in the actively treated group in the per-protocol analysis, prothrombin times were obtained shortly before or within 1 month 


\begin{tabular}{|c|c|c|c|c|c|c|}
\hline & \multicolumn{2}{|c|}{ Sixty-Plus } & \multirow[b]{2}{*}{$\operatorname{HR}(95 \%$ CI $)$} & \multicolumn{2}{|c|}{ WARIS } & \multirow[b]{2}{*}{$\mathrm{RR}(95 \% \mathrm{CI})$} \\
\hline & Anticoagulant & Placebo & & Anticoagulant & Placebo & \\
\hline No. of patients & 439 & 439 & & 607 & 607 & \\
\hline Patient-years (py) of follow-up & 878 & 878 & & 1,872 & 1,872 & \\
\hline Stroke & $12(1.4 / 100 \mathrm{py})$ & $20(2.3 / 100$ py $)$ & $0.60(0.30 ; 1.21)$ & $20(1.1 / 100 \mathrm{py})$ & $44(2.4 / 100$ py) & $0.45(0.23 ; 0.76)$ \\
\hline Intracranial bleeding & 9 & 1 & & 6 & 0 & \\
\hline Fatal & 5 & 1 & & 4 & 0 & \\
\hline Nonfatal & 4 & 0 & & 2 & 0 & \\
\hline Intracranial infarction & 2 & 13 & & 14 & 44 & \\
\hline Fatal & 1 & 5 & & 0 & 10 & \\
\hline Nonfatal & 1 & 8 & & 14 & 34 & \\
\hline Unspecified & 1 & 6 & & - & - & \\
\hline Fatal & 0 & 0 & & & & \\
\hline Nonfatal & 1 & 6 & & & & \\
\hline
\end{tabular}

WARIS $=$ Warfarin Reinfarction trial; $\mathrm{HR}=$ hazard ratios; $\mathrm{CI}=$ confidence intervals; $\mathrm{RR}=$ rate ratios.

prior to the stroke in 17 and 21 patients, respectively. Of the 14 patients with intracranial bleeding, 6 were within the INR range of 3.0 to 4.0 and 8 had an INR of $>4.0$. No intracranial bleedings occurred between INR 2.0 and 3.0. Of the 7 with nonhemorrhagic strokes, 2 had an INR of $<2,3$ had an INR of 3.0 to $4.0,1$ had an INR of $>4.0$, and no measurement was available in 1 patient.

\section{Discussion}

These results indicate and confirm that long-term anticoagulant therapy substantially reduces the risk of stroke in post-myocardial infarction patients. Although the treatment was associated with increased risk of bleeding complications, this was offset by a marked reduction of ischemic events.

A comparison of strokes observed in the two most recently published secondary prevention trials is given in Table 4. The "Sixty-Plus" reinfarction trial randomly allocated patients already treated with oral anticoagulant therapy to continuation (439 patients) or discontinuation (439 patients) of treatment $[2,9]$. The Warfarin Reinfarction trial (WARIS) randomized 1,214 patients with a recent myocardial infarction to either anticoagulant therapy or placebo [10]. The incidence of stroke in these as well as the current trial was reduced by $40 \%$, to $55 \%$, in favor of the anticoagulant group. The incidence in the anticoagulated patients was lowest ( 0.7 per 100 patient-years) in the ASPECT population and highest (1.4/100 patient-years) in the Sixty-Plus trial. The incidence of stroke with placebo was similar in both the Sixty-Plus and WARIS (2.3/ 100 patient-years) trials and was again lowest in the ASPECT group (1.2/100 patient-years). In the SixtyPlus trial, a substantial, higher number of deaths was observed compared with the other two studies. This may be attributed to both the selection of patients as well as new treatment modalities, including thrombolytic therapy and increased frequency of revasculariza- tion in patients with myocardial infarction in the more recent ASPECT trial.

In all three trials, intracranial bleeding occurred more frequently in the anticoagulated group than in the placebo group. The rate of hemorrhagic stroke ( $\mathrm{fa}-$ tal or nonfatal) in the anticoagulated group was $0.3 /$ 100 patient-years in both the WARIS and the ASPECT trials but higher (1.0/100 patient-years) in the Sixy-Plus trial. For placebo-treated patients, the risk was lowest in the WARIS trial ( 0 patients), amounted to $0.04 / 100$ patient-years in the ASPECT trial, and was again highest $(0.1 / 100$ patient-years) in the SixtyPlus trial.

A total of 35 hemorrhagic strokes were observed in the three trials, ie, 32 in the anticoagulated group, of which 17 were fatal, and 3 in the placebo group, of which 1 was fatal. The risk of hemorrhagic stroke observed in the three trials in the treated group was therefore $0.4 / 100$ patient-years against $0.04 / 100$ parientyears in the placebo group. Thus, the incidence of hemorrhagic stroke in the anticoagulated patients was 10 times higher than in untreated patients. Furthermore, about $50 \%$ of the hemorrhagic strokes were fatal. In two population studies conducted in The Netherlands, a similar 8- to 10 -times increase in the risk of intracranial hemorrhage in long-term anticoagulated parients older than 50 years, compared with the total population, was reported $[4,6]$, with at least $50 \%$ of these being fatal $[2-4,6,7]$.

Ischemic strokes (excluding the category unspecified) occurred more frequently in the placebo group than in the anticoagulated group. Fatal ischemic stroke occurred more often in untreated patients than in treated patients in two of the three studies $[2,10]$ and was equally frequent in both treatment groups in the present trial. A total of 31 cerebral infarctions occurred in the three trials in the anticoagulated group compared with 100 in the placebo group. A combined incidence of $0.4 / 100$ patient-years in the treated group and of $1.3 / 100$ patient-years in the placebo group was ob- 
served. This finding indicates that untreated patients have at least a threefold increased risk of ischemic stroke compared with treated patients. Therefore, despite a 10 -fold risk of bleedings in anticoagulated patients, the overall outcome of patients treared with anticoagulation was better than with placebo. In patients on anticoagulants, 18 patients who suffered a stroke had either died or were moderately to severely disabled, against 27 in the placebo group in the current ASPECT trial.

Intracranial bleeding has been reported to occur more frequently with increased $[3,6,18-24]$, and infarction with lower $[2,22-24]$, anticoagulant intensities. The therapeutic range for the two Dutch trials was 2.5 to $5.0 \mathrm{INR}$. In the present study, no intracranial bleedings occurred at INR intensities between 2.0 and $3.0,6$ bleeds occurred berween INR 3.0 and 4.0 , and 8 occurred at intensities above 4.0 INR. These findings extend the previous observations and indicate that, for reasons of safety, the intensity of anticoagulant therapy should not exceed INR 4.0.

Some elements of the current trial and its analysis deserve particular attention. First, ASPECT was a placebo-controlled trial, and the described results are therefore not indicative of the effects of oral anticoagulation relative to treatment with aspirin. Unfortunately, as of yet, there are no large trials in post-myocardial infarction patients that have compared the two treatment modalities directly, and the answrer to the relative efficacy of these treatment options must await results of currently ongoing trials. Although most cardiologists, in the United States as well as in Europe, prefer the easy-to-use aspirin over the frequent monitoring associated with anticoagulant therapy, indirect evidence suggests that the anticoagulant agents may be better. Pooled results of the Antiplatelet Trialists group showed a $12 \%$ reduction in mortality, $31 \%$ reduction in myocardial infarction, and $39 \%$ reduction in strokes [25]. Pooled results of anticoagulant trials showed a $19 \%$ mortality reduction, $44 \%$ reduction in both myocardial infarction and in stroke [24]. Indirect support for better effectiveness is also obtained from findings in patients with atrial fibrillation where oral anticoagulation was generally found to be more effective than aspirin in preventing stroke $[26,27]$.

Some limitations of our study should be mentioned. As a result of its design, information gathered following hospital discharge was limited to clinical events, and we were therefore unable to assess the influences of changes in, for instance, smoking habits or blood pressure during follow-up that could have modified the subsequent risk for stroke. For the same reason, we were unable to speculate on the relation of the cardiac status and the risk for thromboembolic stroke. However, since $95 \%$ of the patients were without evidence of heart failure at randomization, we must assume that most thromboembolic complications were not associated with specific baseline cardiac abnormalities. Another limitation concerns the analysis of the CT scans, which were not centrally reviewed; ie, no distinction could be made between intracerebral hemorrhage and hemorrhagic infarction. Although no patients were lost to follow-up, $30 \%$ of our patients discontinued prematurely trial medication. There were two reasons for this relatively high dropout rate. First, the duration of the trial (6 years) was longer than anticipated, which prompted the refusal of patients to continue trial medication. Second, with the upcoming popularity of aspirin during the course of the trial, most patients who survived a vascular event were subsequently treated with oral anticoagulant therapy or aspirin. This also explains the modest reduction in mortality as observed in this trial. However, we do not believe that the validity of our results was effected by the dropout rate. This is attested by the results of the data, which were analyzed using both the intention-to-treat and the perprotocol analysis, both yielding similar results.

In conclusion, long-term anticoagulant therapy substantially reduced the risk of stroke in post-myocardial infarction patients. Although the rate of intracranial bleeding, the most serious complication of anticoagulant therapy, was increased at least 10-fold, this was offset by a marked reduction in ischemic events of comparable functional outcome. The risk of intractanial bleeding was directly related to the intensity of anticoagulant treatment. Approximately $60 \%$ of the hemorrhagic strokes occurred at an INR of $>4$, and this value should therefore be considered the upper limit for safe oral anticoagulant therapy.

\section{References}

1. Jonker JJC, Chesebro JH. Chronic coronary disease. In: Fuster $V$, Verstraete $M$, eds. Thrombosis in cardiovascular disorders. Philadelphia: WB Saunders, 1992:363-374

2. Sixty Plus Reinfarction Study Research Group. Risks of longterm oral anticoagulant therapy in elderly patients after myocardial infarction. Lancet 1982;1:64-68

3. Fogelholm R, Eskola K, Kiminkinen T, Kunnamo I. Anticoagulant treatment as a risk factor for primary intracerebral haemorrhage. J Neurol Neurosurg Psychiary 1992;55:1121-1124

4. Franke CL, de Jonge $\mathrm{H}$, van Swieten JC, et al. Intracerebral hematomas during anticoagulant treatment. Stroke 1990;21: $726-730$

5. Foulkes MA, Wolf PA, Price TR. The stroke data bank: design, methods, and baseline characteristics. Stroke 1988;19:547-554

6. Wintzen AR, de Jonge H, Loeliger EA, Bots GTAM. The risk of intracerebral hemorrhage during oral anticoagulant treatment: a population study. Ann Neurol 1984;16:553-558

7. Meade TW, ed. Anticoagulant and myocardial infarction: a reappraisal. New York: John Wiley, 1984

8. Anticoagulants in the Secondary Prevention of Events in Coronary Thrombosis (ASPECT) Research group. Effect of longterm anticoagulant treatment on mortality and cardiovascular morbidity after myocardial infarction. Lancet 1994;1:499-503

9. Sixty Plus Reinfarction Study Research Group. A double-blind 
trial to assess long-term oral anticoagulant therapy in elderly patients after myocardial infarction. Lancet 1980;2:989-993

10. Smith P, Arnesen H, Holme I. The effect of warfarin on mortality and reinfarction after myocardial infarction. $N$ Engl $J$ Med 1990;323:147-152

11. Loeliger EA. The optimal therapeutic range in oral anticoagulation. History and proposal. Thromb Haemost 1979;42:11411152

12. Loeliger EA, Broekmans AW. Optimal therapeutic anticoagulation. Haemostasis 1985;15:283-292

13. Loeliger EA, yan den Besselaar AMHP, Broekmans AW. Intensity of oral anticoagulation in patients monitored with various thromboplastins. N Engl J Med 1983;1:1228 (Letter)

14. Loeliger EA. Laboratory control, optimal therapeutic ranges and therapeutic quality control in oral anticoagulation. Acta Haematol (Basel) 1985;74:125-131

15. Loeliger EA. Therapeutic target values in oral anticoagulation-justification of Dutch policy and a warning against the so-called moderate-intensity regimens. Ann Hematol 1992;64: $60-65$

16. The Ad Hoc Committee on the classification and outline of cerebrovascular disease, Il (Chairman CH Milikan). Stroke $1975 ; 6: 566-616$

17. Cox DR. Regression models and life tables. J R Stat Soc [B] 1972;34:187-220

18. Levine MN, Raskob G, Hirsh J. Hemorrhagic complications of long-term anticoagulant therapy. Chest 1986;89(Suppl): $16 S-25 S$

19. Levine MN, Raskob G, Hirsh J. Hemorrhagic complications of long-term anticoagulant therapy. Chest 1989;95(Suppl): $26 \mathrm{~S}-36 \mathrm{~S}$

20. Landefeld CS, Rosenblatt MW, Goldman L. Bleeding in outpatients treated with warfarin: relation to the prothrombin time and important remediable lesions. Am J Med 1989;87: 153-159

21. Launbjerg J, Egeblad H, Heaf J. Bleeding complications to oral anticoagulant therapy: multivariate analysis of 1010 treatment years in 551 outpatients. J Int Med 1991;229:351-355

22. van der Meer FJM, Rosendaal FR, Vandenbroucke JP, Briët E. Blceding complications in oral anticoagulant therapy: an analysis of risk factors. Arch Intern Med 1993;153:15571562

23. Rosendaal FR, Cannegieter SC, van der Meer FJM, Briët E. A method to determine the optimal intensity of oral anticoagulant therapy. Thromb Haemost 1993;69:236-239

24. Azar AJ. Therapeutic achievement with long-term oral anticoagulants in post-myocardial infarction patients. Dissertation, Erasmus University Rotterdam, The Netherlands, 1993

25. Antiplatelet Trialists' Collaboration. Collaborative overview of randomised trials of antiplatelet therapy-l: Prevention of death, myocardial infarction, and stroke by prolonged antiplatelet therapy in various categories of patients. Br Med J 1994;308:81-101

26. European Atrial Fibrillation Trial Study Group. Secondary prevention in non-rheumatic atrial fibrillation after transient ischemic attack or minor stroke. Lancet 1993;342:1255-1262

27. Albers GW. Atrial fibrillation and stroke. Arch Intern Med 1994;154:1443-1448 